\title{
RESONANCE FLUORESCENCE SPECTRUM OF TWO-LEVEL ATOMS DRIVEN BY TWO NONCOLLINEAR CLASSICAL FIELDS
}

\author{
B. ŁoBODZIŃSKI \\ Instytut Fizyki, Uniwersytet Jagielloński \\ Reymonta 4, 30-059 Ḱraków, Poland
}

(Received April 29, 1997)

The resonance fluorescence spectrum of an ensemble of two-level atoms driven by two classical, frequency degenerate and noncollinear laser beams is investigated. It has been found that the spatially-averaged resonance fluorescence spectra differ significantly from the spectra of two-level atoms calculated for the single-beam excitation. The diflerences were noticed in the number of peaks, their positions and shapes and in their dependences on an angle between wave vectors of the incidcnt beams.

PACS numbers: $32.50 .+\mathrm{d}, 42.50 . \mathrm{II} z, 42.25 . \mathrm{Iz}$

Resonance fluorescence of two-level atoms interacting with a colherent field has been the subject of numerous studies [1]. It was shown that modifications of a fluorescence spectrum (FS) may be affected by many reasons, for example: squeezed reservoir [2], non-Markovian processes [3], collisions [4, 5] and a chaotic or Gaussian statistics of the incident field [6]. Only the last two features affect the spectra in a free space [5], the former require cavities. One interesting problem is the FS of two-level atoms driven by more than one external fields. Two model situations are of fundamental interest here:

(1) two collinear, coherent ficlds with different frequencies (bichromatic excitation),

(2) two noncollinear, coherent fields with the same frequencies (noncollinear excitation).

FS of a two-level atom driven by bichromatic fields is now well understood [7]. The second case, FS of an ensemble of two-level atoms driven by noncollinear beams, is the subject of this paper. We assume that the density of our atomic system is so small that we can neglect collisions.

We consider a physical situation where the ensemble of two-level atoms is placed in the intersection of two cohercnt laser fields with the same frequency $\omega_{L}$ and different wave vectors $k_{1}$ and $k_{2}$. $\Lambda$ scheme of such an experiment is sketched in Fig. 1. The objective is to calculate the stationary fluorescence spectrum of the ensemble of two-level atoms as a function of angle 0 between the wave vectors of the driving laser beams. 


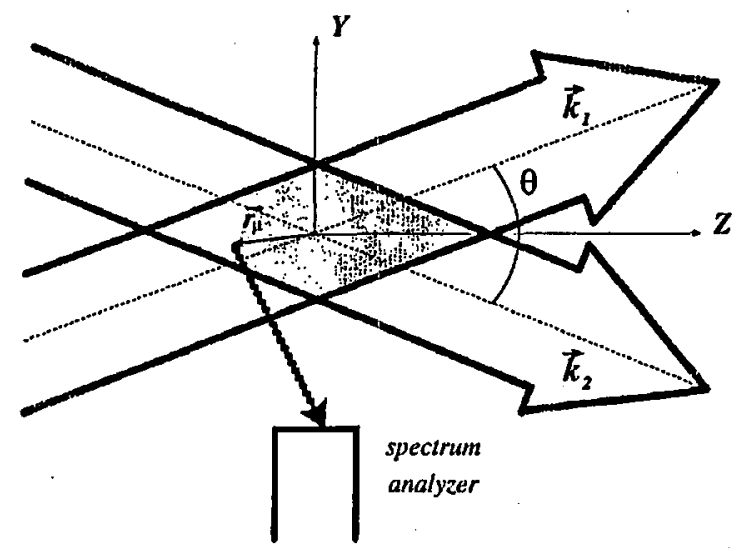

Fig. 1. The considered geometry: two-level atoms are placed within the intersection of two coherent laser beams with wave vectors $k_{1}$ and $k_{2}$. Both fields have the same frequency $\omega_{\mathrm{L}}$. The fluorescence spectrum is registered by a spectrum analyzer.

The driving field is

$$
\boldsymbol{E}(t, \boldsymbol{r})=\left\{\boldsymbol{E}_{1} \exp \left[-\mathrm{i}\left(\omega_{\mathrm{L}} t-\boldsymbol{k}_{1} \cdot \boldsymbol{r}\right)\right]+\boldsymbol{E}_{2} \exp \left[-\mathrm{i}\left(\omega_{\mathrm{L}} t-\boldsymbol{k}_{2} \cdot \boldsymbol{r}\right)\right]\right\}+\text { c.c. }
$$

where $\boldsymbol{E}_{1}$ and $\boldsymbol{E}_{2}$ are the amplitudes of two incident fields. Denoting Rabi frequencies of the driving fields by

$$
\Omega_{1}=d \cdot E_{1}, \quad \Omega_{2}=d \cdot E_{2},
$$

where $d$ is the atomic electric dipole moment and we put $\hbar=1$, we can write the total Rabi frequency in the following form:

$$
\Omega(r)=\Omega_{1} \exp \left(\mathrm{i} k_{1} \cdot r\right) f(r, \theta) .
$$

Here the following variables are introduced:

$$
f(r, \theta)=1+\chi \exp [\mathrm{i} \varphi(r, \theta)], \quad \chi=\Omega_{2} / \Omega_{1}, \quad \varphi(r, \theta)=\left(k_{2}-k_{1}\right) \cdot r,
$$

$f(r, \theta)$ could be treated as a spatially-dependent coupling constant. The resulting Hamiltonian of our system in the rotating-wave approximation can be written as

$$
H=\sum_{\mu}\left\{\Delta_{L} \sigma_{3 \mu}+\frac{\mathrm{i}}{2} \Omega_{1}\left[f\left(r_{\mu}, \theta\right) \sigma_{\mu}^{+}-f^{*}\left(\boldsymbol{r}_{\mu}, \theta\right) \sigma_{\mu}\right]\right\},
$$

where $\Delta_{\mathrm{L}}=\omega_{0}-\omega_{\mathrm{L}}$ (with $\omega_{0}$ being the atomic transition frequency), $\sigma_{\mu}^{+}, \sigma_{3 \mu}$ are the usual atomic operators describing the $\mu$-th atom, $\sigma_{3 \mu}=|2\rangle\left\langle\left. 2\right|_{\mu}-\mid 1\right\rangle\left\langle\left. 1\right|_{\mu}\right.$, $\sigma_{\mu}^{+}=|2\rangle\left\langle\left. 1\right|_{\mu}, 1\right.$ and 2 refer to the lower and upper state, respectively, and $r_{\mu}$ describes the position of the $\mu$-th atom. Since the density of the considered ensemble is small (no collisions) it is sufficient to introduce only the radiative atomic relaxation (with rate $\gamma$ ) to the master equation for the $s$-th atom,

$$
\frac{\partial}{\partial t} \rho_{s}=\mathrm{i}\left[H, \rho_{s}\right]+\gamma\left(2 \sigma_{s} \rho_{s} \sigma_{s}^{+}-\sigma_{s}^{+} \sigma_{s} \rho_{s}-\rho_{s} \sigma_{s}^{+} \sigma_{s}\right) \text {. }
$$

As has been shown, e.g. in Ref. [8], the positive-frequency part of the electric field produced by an atom at some position $r_{\mu}$ in the far-field limit is

$$
E_{\mu}^{(+)}\left(r_{\mathrm{d}}, t\right)=g_{\mu} \sigma_{\mu}^{+}\left(t-\frac{\left|r_{\mathrm{d}}-r_{\mu}\right|}{c}\right)
$$


where $r_{\mathrm{d}}$ is the position of the detector and the geometrical factor $g_{\mu}$ is expressed in the following form:

$$
g_{\mu}=\frac{\omega_{0}^{2}}{4 \pi \epsilon_{0} c^{2}}\left\{\frac{d}{\left|r_{\mathrm{d}}-r_{\mu}\right|}-\frac{\left[d\left(r_{\mathrm{d}}-r_{\mu}\right)\right]\left(r_{\mathrm{d}}-r_{\mu}\right)}{\left|r_{\mathrm{d}}-r_{\mu}\right|^{2}}\right\} .
$$

Assuming that the linear dimensions of the scattering volume are small in comparison with the distance between the scattering volume and the detector we may neglect the dependence of the geometrical factor on the position of the atom and take $g_{\mu} \approx g$.

The FS was calculated using the quantum regression theorem [1]. In general, the FS for the considered geometry can be represented as a sum of two components

$$
S(\omega)=S_{1}(\omega)+S_{2}(\omega)
$$

where $S_{1}(\omega)$ describes the two-atom correlation function

$$
\begin{aligned}
& S_{1}(\omega)=|g|^{2} \int_{-\infty}^{+\infty} \mathrm{d} \tau \\
& \times\left\{\sum_{s=1}^{N} \sum_{l=1, l \neq s}\left\langle\sigma_{l}^{+}(\tau) \sigma_{s}(0)\right\rangle \exp \left[-\mathrm{i}\left(k-k_{1}\right)\left(r_{l}-r_{s}\right)\right]\right\} \exp \left[\mathrm{i}\left(\omega-\omega_{\mathrm{L}}\right) \tau\right]
\end{aligned}
$$

with $k$ being the wave vector in the direction of the detector. Because the cooperative effects are negligibly small, i.e. the atoms are sufficiently far from each other and have random positions, $S_{1}(\omega)$ tends to zero for sufficiently large $N$. The second term $S_{2}$ represents the single-atom correlation and

$$
S_{2}(\omega)=|g|^{2} \int_{-\infty}^{+\infty} \mathrm{d} \tau\left[\sum_{s=1}^{N}\left\langle\sigma_{s}^{+}(\tau) \sigma_{s}(0)\right\rangle\right] \exp \left[\mathrm{i}\left(\omega-\omega_{\mathrm{L}}\right) \tau\right] .
$$

IIence, the resulting FS of a large number of atoms placed in the intersection of the driving fields is simply a sum of the fluorescence spectra of independent atoms. Since the atoms are distributed randomly, the FS does not exhibit intrinsic quantum fluctuations leading to the phenomenon of antibunching [9]. However, it can be modified by a classical interference of two intersecting laser beams. This interference appears in the generalized Rabi frequency for the $\mu$-th atom as a term proportional to $\cos \left(\varphi\left(\boldsymbol{r}_{\mu}, \theta\right)\right)$

$$
\Omega_{\mu}=\left\{\Omega_{1}^{2}\left[1+2 \chi \cos \left(\varphi\left(r_{\mu}, \theta\right)\right)+\chi^{2}\right]+\Delta_{\mathrm{L}}^{2}\right\}^{1 / 2} .
$$

In that way, our problem could be simplified to the calculations of FS of individual atoms placed in the spatially inhomogeneous external field. FS of each atom is the Mollow triplet with the side band separation characterized by the local Rabi frequency which, according to Eq. (11), depends on the oscillating term $2 \Omega_{1}^{2} \chi \cos \left(\varphi\left(r_{\mu}, \theta\right)\right)$. Therefore, two extreme positions of the side bands are determined by the extreme values of the generalized Rabi frequency

$$
\Omega_{\max }=\left[\Omega_{1}^{2}\left(1+2 \chi+\chi^{2}\right)+\Delta_{\mathrm{L}}^{2}\right]^{1 / 2}
$$

and

$$
\Omega_{\min }=\left[\Omega_{1}^{2}\left(1-2 \chi+\chi^{2}\right)+\Delta_{L}^{2}\right]^{1 / 2} .
$$


The behaviour of the positions of the side bands within the region determined by $\Omega_{\max }$ and $\Omega_{\min }$ was analyzed numerically. For simplicity, additional assumptions were made:

(i) The beams are plane waves in the $Y Z$ plane (Fig. 1). This could be practically accomplished also with the Gaussian beams in the central part of their intersection.

(ii) The detector (spectrum analyzer) records the FS only from these atoms that are distributed in the intersection of the incident beams, i.e. a possible background of atoms excited by single beams is ignored.

For the atom at position $r_{\mu}$ the spatial phase $\varphi\left(r_{\mu}, \theta\right)$, introduced in Eq. (4) is

$$
\varphi\left(r_{\mu}, \theta\right)=\left(k_{2}-k_{1}\right) \cdot r_{\mu}=A_{\mu} \cos \left(\alpha_{\mu}\right) \sin (\theta / 2),
$$

where $A_{\mu}=2\left|k_{1}\right|\left|r_{\mu}\right|\left(\left|k_{1}\right|=\left|k_{2}\right|=2 \pi / \lambda, \lambda\right.$ being the wavelength $)$ and $\alpha_{\mu}$ is the angle between $k_{1}$ and $r_{\mu}$.

Since the positions of atoms are random, the product $A_{\mu} \cos \left(\alpha_{\mu}\right)$ can be expressed using random numbers as

$$
A_{\mu} \cos \left(\alpha_{\mu}\right)=\max \left\{A_{\mu}\right\} \eta_{1} \cos \left(2 \pi \eta_{2}\right)
$$

where $\eta_{1}$ and $\eta_{2}$ are independent random numbers from the normal interval $[0,1]$. Product $\max \left\{A_{\mu}\right\} \eta_{1}$ plays the role of the $2\left|k_{1}\right|\left|r_{\mu}\right|$ value and $2 \pi \eta_{2}$ is the random angle $\alpha_{\mu}$. The value of $\max \left\{A_{\mu}\right\}$ was estimated as follows. Assuming that $\lambda \approx 600 \mathrm{~nm}$ and the maximum dimension of the beam-crossing area is $\max \left\{\left|r_{\mu}\right|\right\} \approx 0.01 \mathrm{~m}$, get

$$
\max \left\{A_{\mu}\right\}=2\left|k_{1}\right|\left|r_{\mu}\right|=4 \pi \frac{\max \left\{\left|r_{\mu}\right|\right\}}{\lambda} \approx 10^{5},
$$

therefore, as can be seen from Eq. (14), the influence of the interference term in $\Omega_{\mu}$ (Eq. (11)) is maximal if spatial phase $\varphi\left(r_{\mu}, \theta\right)$ oscillates around 0 , i.e. when the angle between the laser beams is sufficiently small.

Under the discussed conditions, the cflect of the two-beam interference on FS is also dependent on the laser detuning $\Delta_{\mathrm{L}}$. In our case, this effect is maximal for $\Delta_{L}=0$ and decreases when $\left|\Delta_{\mathrm{L}}\right|$ is growing. More complicated is the dependence of the interference phenomena on $\chi$ and 0 . For better determination of the visibility of the modulational term in Eq. (11), let us define the contrast parameter $\epsilon(\chi, \theta)$ as

$$
\epsilon(\chi, \theta)=\left|\frac{2 \chi \sum_{\mu=1}^{N} \cos \left(\varphi\left(r_{\mu}, 0\right)\right)}{1+\chi^{2}}\right| .
$$

The dependence of $\epsilon$ on $\chi$ and $\theta$ is shown in Fig. 2. $\epsilon$ reaches its maximum value when both beams have the same Rabi frequency $(\chi=1)$ and when $\theta=0$. This case is equivalent to a simple one-beam excitation with the Rabi frequency $\Omega=$ $\left.\Omega_{\max }\right|_{\Delta_{L}=0, X=1}=2 \Omega_{1}$. For $\theta>0$, however, additional maxima of $\epsilon$ appear. Their effect on FS depends on the ratio of their amplitudes to the amplitude of $\left.\epsilon\right|_{\theta=0}$ for a given $\chi$. The highest amplitudes of the fluorescence side bands can be observed for $\chi=1$. The spectrum calculated as a function of 0 for this case is shown in Fig. 3a. The spectrum is symmetric around $\omega_{\mathrm{L}}$ so only its one wing, $\omega-\omega_{\mathrm{L}}>0$, is shown and the central component of FS, normalized to 1 , is cut for better 


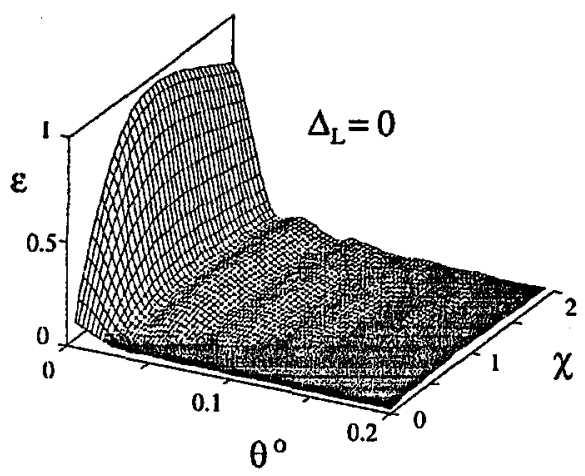

Fig. 2. Dependence of the contrast parameter $c(\chi, \theta)$ on angle $\theta$ between the wave vectors and the ratio of the Rabi frequencies $\chi$, calculated for $\Delta_{\mathrm{L}}=0$. Values of $\theta$ are given in degrees.
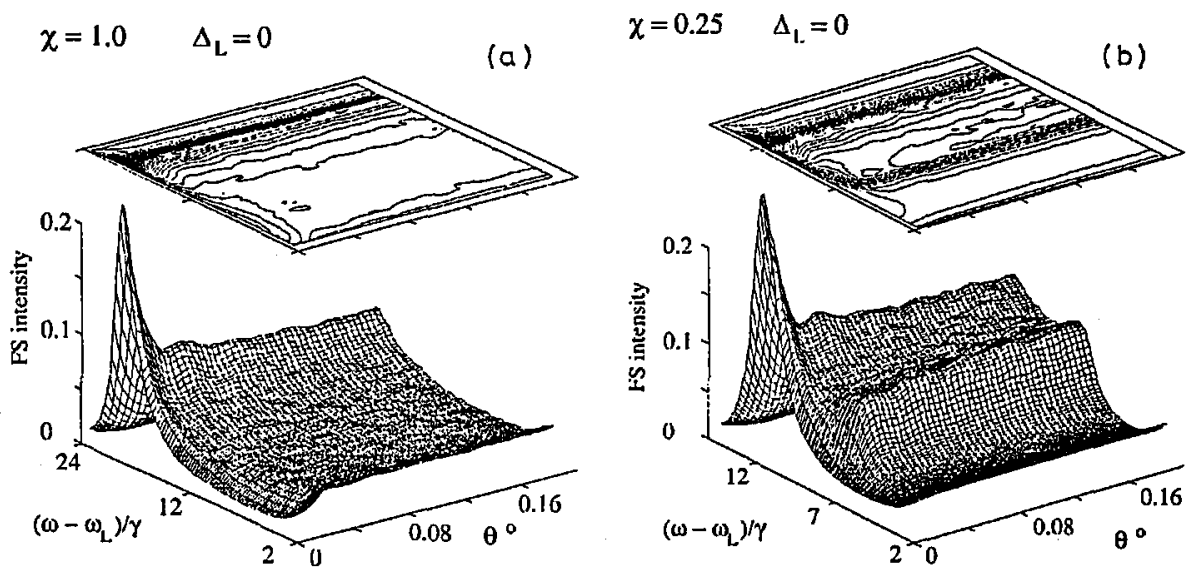

Fig. 3. The resonance fluorescence spectra for the Rabi frequency $\Omega_{1} / \gamma=10$ and for $x=1$ (a) and $x=0.25$ (b) as a function of the angle between the wave vectors of the incident beams $\theta$ and $\left(\omega-\omega_{\mathrm{L}}\right) / \gamma$. In both cases the laser detuning $\Delta_{\mathrm{L}}=0$. The spectra are normalized to the amplitude of the central component $\left(\omega=\omega_{\mathrm{L}}\right)$ and to the number of drew lots positions of atoms (number of atoms $N=1000$ ) and are symmetrical around the laser frequency. Above each spectrum, its projection in a form of a contour-plot is shown.

visualization of the modulation effects. As seen, the spectrum has the form of a standard triplet and the two beam-interference manifests itself by oscillating amplitude of the side band. With increasing $\theta$, interference behaviour of FS as a function of $\theta$ is suppressed. Since in this case $\Omega_{\min }=0$, FS is perturbed by the interference part in the whole range of $\omega-\omega_{\mathrm{L}}$. Another particularly interesting behaviour of FS may be observed for parameters: $\chi=0.25$ and $\Delta_{\mathrm{L}}=0$. In this case, we have $\Omega_{\min }=0.75 \Omega_{1}$ and $\Omega_{\max }=1.25 \Omega_{1}$. The resulting FS is shown in Fig. $3 \mathrm{~b}$. One can see that side bands acquire an extra oscillating structure for such 
angles $\theta$ for which the contrast parameter $\epsilon$ reaches its minima.

As seen from Fig. 2, the differences between successive extrema of the contrast parameter disappear with increasing $\theta$. For a big angle $\theta$ and $\Delta_{\mathrm{L}}=0$, the generalized Rabi frequency can be approximated by

$$
\Omega_{\mu}=\Omega_{1}\left[1+2 \chi \cos \left(\varphi\left(r_{\mu}, \theta\right)\right)+\chi^{2}\right]^{1 / 2} \Rightarrow \Omega_{1}\left(1+\chi^{2}\right) .
$$

So, in this way, FS is independent of $\theta$. The FS recorded for the perpendicular or counter-propagating beams does not show any oscillations versus $\theta$.

Summarizing, fluorescence spectrum of the diluted sample of two-level atoms, driven by two noncollinear laser beams, exhibits oscillations resulting from the classical interference of incident fields. This novel feature results from averaging over positions of independent atoms in the inhomogeneous field within the two-beam intersection. The oscillating nature of FS disappears when the laser detuning and the angle between beams increase. It is also negligible for small values of the ratio of the Rabi frequencies of the incident fields $(\chi)$. The periodic dependence of FS on the angle between the beams depends on the resonant frequency of atoms. If $\omega_{0}$ decreases then the range of the angles for which the described effect occurs is wider. Analogous, similar behaviour could be expected for more frequency degenerate, and noncollinear laser beams and could be observed, e.g. in the ensemble of atoms trapped with several intersecting beams [10].

The author is grateful to W. Gawlik, E. Mroczko, G. Wasik, J. Zachorowski and J. Zakrzewski for their remarks on this paper. The work was supported by the Committee for Scientific Research (grant 2P03B-113-10) and by the EU grant (COST:CIPA-CT93-0094).

\section{References}

[1] B.R. Mollow, Phys. Rev. 188, 1969 (1969); see also a review on resonance fluorescence: P.L. Knight, P.W. Milonni, Phys. Rep. 66, 21 (1980).

[2] H.J. Carmichael, A.S. Lane, D.F. Walls, Plıys. Rev. Lett. 58, 2539 (1987).

[3] K. Wódkiewicz, J.H. Eberly, Ann. Phys. (N.Y.) 101, 574 (1976); M. Lewenstein, T.W. Mossberg, R.J. Glauber, Phys. Rev. Lett. 59, 775 (1987).

[4] B.R. Mollow, Phys. Rev. A 13, 758 (1976).

[5] J.L. Carlsten, A. Szöke, M.G. Raymer, Phys. Rev. A 15, 1029 (1977).

[6] P. Zoller, in: Multiphoton Processes, Eds. P. Lambropoulos, S.J. Smith, Vol. 2, Springer-Verlag, Berlin 1984, p. 68 and references therein.

[7] Z. Ficek, H.S. Freedhoff, Phys. Rev. A 48, 3092 (1993) and references therein.

[8] G.S. Agarwal, Quantum Optics, Ed. G. Höhler, in series Springer Tracts in Modern Physics, Vol. 70, Springer-Verlag, Berlin 1974; H.J. Kimble, L. Mandel, Phys. Rev. A 13, 2123 (1976).

[9] R. Loudon, Opt. Commun. 49, 24 (1984).

[10] P. Verkerk, D.R. Meacher, A.B. Coates, J.-Y. Courtis, S. Guibal, B. Lounis, C. Salomon, G. Grynberg, Europhys. Lett. 26, 171 (1994). 